\title{
How to Approach a Patient with Eosinophilia
}

\author{
Luis Villela ${ }^{1}$ and Vittorio García-Zamudio ${ }^{2}$ \\ ${ }^{1}$ Hematology Service and Blood Bank; 'Internal Medicine Service. "Dr. Ignacio Chávez" Medical Center, ISSSTESON, Hermosillo. Sonora, Mexico
}

\begin{abstract}
Eosinophilia is an increase in the absolute number of eosinophils (> 500 absolute number). It can be divided into two major groups: secondary eosinophilia (the most common) and primary eosinophilia (not common). This article will focus on providing the personal opinion of the authors about how we approach eosinophilia in the "Dr. Ignacio Chávez" Medical Center of ISSSTESON, in a way comprehensible to both the medical student and the subspecialist. This article will not focus on treatment since treatment will depend on each nosological entity.
\end{abstract}

Key words: Eosinophilia. Hypereosinophilia. Diagnostic. Approach.

\section{Introduction}

Eosinophilia is a finding that is not uncommon to see in patients, both in outpatients and in those admitted to a hospital. When this finding comes with very "obvious" signs and symptoms, it is very useful to obtain clues and start to treat those patients. There is a large variety of disorders that can be linked to peripheral eosinophilia. These entities range from parasitic helminth infection to neoplastic processes ("simply" the expression of a hidden or non-hidden neoplasia).

It is important to understand the role that the eosinophil plays in the human body. In doing so, we will be able to understand the clinical expression with which the different entities are presented. There are two essential interleukins for the maturity of eosinophils: interleukin-5 (IL-5) and interleukin-3 (IL-3). These cytokines, in conjunction with the granulocytes and macrophages' growth factor (FEC-GM), are responsible for maturing and setting free the eosinophil from the bone marrow ${ }^{1}$. The actions of the eosinophils vary and are in charge mainly of innate immunity (the defense system we are born with) and inflammation. Liberation of different molecules such as leukotrienes and histamine can occur ${ }^{2}$. In healthy individuals, the eosinophils are located in the lymph nodes, digestive tract (except in the esophagus), thymus, spleen, mammary glands, and uterus $^{1,2}$.

For the approach or diagnosis of eosinophilia, we ought to ask ourselves the following questions:

1. Does my patient really have eosinophilia? The definition of eosinophilia is a number over $0.5 \times 109 / \mathrm{L}$ or a total of 500 eosinophils. Here, I would like to stress that when we evaluate the absolute number, this is obtained by multiplying the percentage of eosinophils by the total number of leukocytes. We must never call it eosinophilia when dealing with just an elevated percentage of eosinophils; it must always be the absolute number! For example, 9000 leukocytes with $7 \%$ eosinophils. The percent value is elevated, but the absolute number is 490 . Hence, it is not eosinophilia. On the other hand, a case

\section{Correspondence:}

Luis Villela

E-mail: I.villela @isssteson.gob.mx CC BY-NC-ND license (http://creativecommons.org/licenses/by-nc-nd/4.0/).
Date of reception: 30-01-2018

Date of acceptance: 30-01-2018

DOI: 10.24875/RMU.M18000007
Available online: $30-05-2018$ Medicina Universitaria. 2018;20(1):55-57 www.medicinauniversitaria.org open access article under the 
with 16,000 leukocytes and 4\% eosinophils, the percent value is normal, but with a mild absolute total eosinophilia count of 640 .

2. What intensity of eosinophilia does my patient have? Hematologists classify the high number of eosinophilia as follows: (a) mild, a total count ranging between 501 and 1499 eosinophils, (b) moderate, between 1500 and 4999 total eosinophils, and (c) severe, over 5000 total number of eosinophils.

3. Does my patient have hypereosinophilia? Hypereosinophilia is considered when the number of eosinophils is $\geq 1500$, that is, between moderate and severe eosinophilia, and the key is that this number is linked to organ or tissue damage, regardless of the underlying cause. An example of this would be the damage to dermal tissue in a Drug reaction with eosinophilia and systemic symptoms syndrome, where the damaged tissue would be the skin (Fig. 1). Here, the activated lymphocytes (TH2 type) play a major role, with a vast secretion of IL-51.

4. Is it primary or secondary hypereosinophilia? Hypereosinophilia can be divided into polyclonal and monoclonal. The most frequently observed is polyclonal hypereosinophilia. In other words, it is secondary or reactive to other known or unknown illnesses which secrete cytokines (IL-3, IL-5, and FEC-GM). Polyclonal or secondary hypereosinophilia is observed in parasite infestations (filaria, Strongyloides stercoralis, Schistosoma spp., and Toxocara species), allergens (medications), and vasculitis (ChurgStrauss syndrome and Wegener granulomatosis). Furthermore, in collagen diseases (systemic erythematosus lupus), myalgia-eosinophilia syndromes (tryptophan ingestion), pulmonary eosinophilic disease (eosinophilic pneumonias, tropical pulmonary eosinophilia, and bronchopulmonary aspergillosis), allergic gastroenteritis (linked to eosinophilia), adrenal insufficiency, solid neoplasms (adenocarcinomas), or hematological neoplasms (chronic and acute myeloproliferative syndromes with eosinophilia, Hodgkin lymphoma, and peripheral T-lymphoma) $)^{3,4}$. Monoclonal or primary hypereosinophilia results from direct eosinophilic expansion, we observe not cytokines, but specific mutations at a stem cell level are found $d^{3,4}$. Here, the specialist (hematologist) approach will be more specific and should be focused on the peripheral evaluation such as the bone marrow where the morphology would be assessed (dysplastic cells, presence of blast cells, and monocytosis), cytometry (tryptase+, CD117+, and CD25+), typical cytogenetics (4q12, 5q31-33, and 8p11-12),

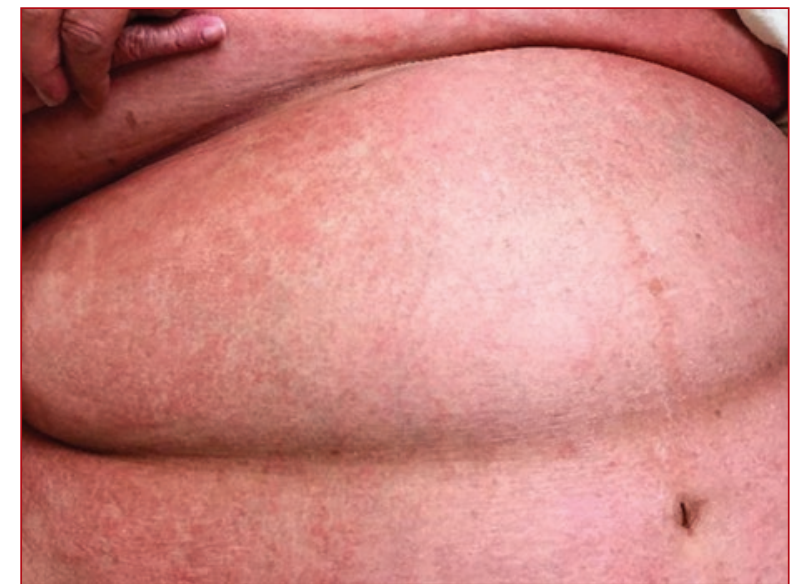

Figure 1. A 68-year-old female patient who was admitted to the "Dr. Ignacio Chavez" Medical Center of ISSSTESON for a general seizure, fever, joint pain, and leukocytes of $6 \times 10^{3} / \mathrm{L}$ with an absolute count of 2230 eosinophils. She had a history of taking medications such as allopurinol, nonsteroidal analgesics (nonsteroidal anti-inflammatory drugs), and ciprofloxacin. A diagnosis of Drug reaction with eosinophilia and systemic symptoms was performed, significantly improving with the use of steroids.

and molecular biology (specific mutations such as FIP1L1-PDGFRA and PCM1-JAK2). It is important to mention that the most frequent mutation seen in monoclonal hypereosinophilia is in the FIP1L1-PDGFRA hybrid gene (95\% of cases). However, not all hospitals have a molecular biology unit which shows results relatively fast. In these cases, serum tryptase correlates very well with the presence of this mutation; nevertheless, it may be increased in myeloproliferative syndromes with eosinophilia ${ }^{5}$.

After answering these four questions, we will be able to have a more accurate approach to eosinophilia, thus establishing the exact diagnosis to offer the proper treatment. We can observe in Figure 2, what we do in our hospital. Every place should have a chat flow for the approach; we think this is a practical way that you could use in your practical life as doctor, general practitioner, or internal medicine.

\section{Ethical disclosures}

Protection of human and animal subjects. The authors declare that no experiments were performed on humans or animals for this study.

Confidentiality of data. The authors declare that they have followed the protocols of their work center on the publication of patient data. 


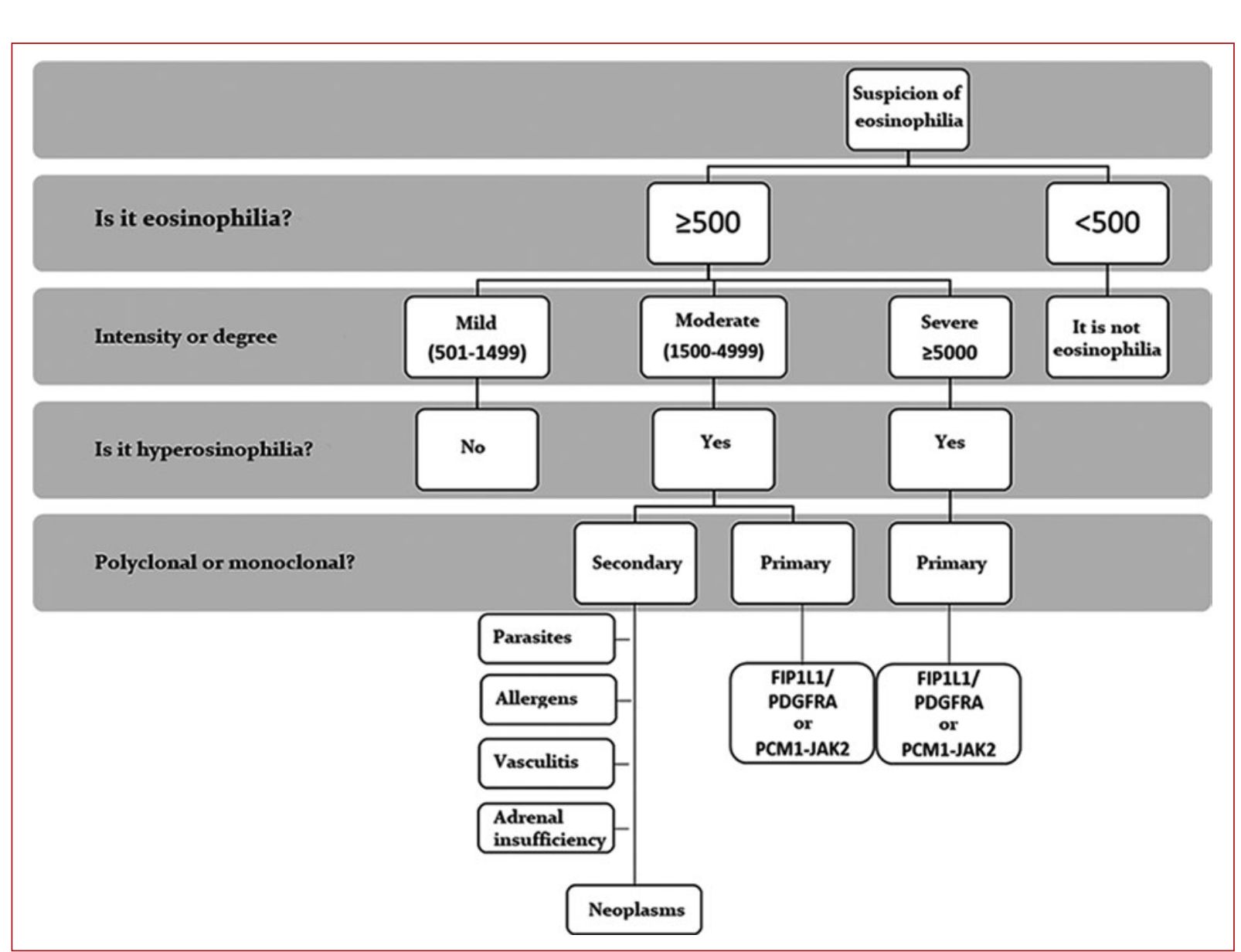

Figure 2. Diagnostic approach of eosinophilia at the "Dr. Ignacio Chávez" Medical Center from ISSSTESON.

Right to privacy and informed consent. The authors have obtained the written informed consent of the patients or subjects mentioned in the article. The corresponding author is in possession of this document.

\section{References}

1. Ackerman SJ, Bochner BS. Mechanisms of eosinophilia in the pathogenesis of hypereosinophilic disorders. Immunol Allergy Clin North Am. 007; 27:357-75
2. Spencer LA, Szela CT, Perez SA, et al. Human eosinophils constitutively express multiple th1, th2, and immunoregulatory cytokines that are secreted rapidly and differentially. J Leukoc Biol. 2009;85:117-23.

3. Tefferi A, Gotlib J, Pardanani A. Hypereosinophilic syndrome and clonal eosinophilia: Point-of-care diagnostic algorithm and treatment update. Mayo Clin Proc. 2010;85:158-64.

4. Gotlib J. World health organization-defined eosinophilic disorders: 2017 update on diagnosis, risk stratification, and management. Am J Hematol. 2017;92:1243-59.

5. Klion $A D$, Noel $P$, Akin $C$, et al. Elevated serum tryptase levels identify a subset of patients with a myeloproliferative variant of idiopathic hypereosinophilic syndrome associated with tissue fibrosis, poor prognosis, and imatinib responsiveness. Blood. 2003;101:4660-6. 